\title{
Weakly and strongly singular solutions of semilinear fractional elliptic equations
}

\author{
Huyuan Chen ${ }^{1}$ \\ Departamento de Ingeniería Matemática \\ Universidad de Chile, Chile \\ Laurent Véron ${ }^{2}$ \\ Laboratoire de Mathématiques et Physique Théorique \\ Université François Rabelais, Tours, France
}

\begin{abstract}
Let $p \in\left(0, \frac{N}{N-2 \alpha}\right), \alpha \in(0,1)$ and $\Omega \subset \mathbb{R}^{N}$ be a bounded $C^{2}$ domain containing 0 . If $\delta_{0}$ is the Dirac measure at 0 and $k>0$, we prove that the weakly singular solution $u_{k}$ of $\left(E_{k}\right)(-\Delta)^{\alpha} u+u^{p}=k \delta_{0}$ in $\Omega$ which vanishes in $\Omega^{c}$, is a classical solution of $\left(E_{*}\right)(-\Delta)^{\alpha} u+u^{p}=0$ in $\Omega \backslash\{0\}$ with the same outer data. When $\frac{2 \alpha}{N-2 \alpha} \leq 1+\frac{2 \alpha}{N}, p \in\left(0,1+\frac{2 \alpha}{N}\right]$ we show that the $u_{k}$ converges to $\infty$ in whole $\Omega$ when $k \rightarrow \infty$, while, for $p \in\left(1+\frac{2 \alpha}{N}, \frac{N}{N-2 \alpha}\right)$, the limit of the $u_{k}$ is a strongly singular solution of $\left(E_{*}\right)$. The same result holds in the case $1+\frac{2 \alpha}{N}<\frac{2 \alpha}{N-2 \alpha}$ excepted if $\frac{2 \alpha}{N}<p<1+\frac{2 \alpha}{N}$.
\end{abstract}

\section{Contents}

1 Introduction $\quad 2$

2 Preliminaries 5

$\begin{array}{lll}3 & \text { Regularity } & 7\end{array}$

4 The limit of weakly singular solutions 9

4.1 The case $p \in\left(0,1+\frac{2 \alpha}{N}\right] \ldots \ldots \ldots \ldots \ldots$

4.2 The case $p \in\left(1,1+\frac{2 \alpha}{N}\right] \ldots \ldots \ldots \ldots \ldots$

4.3 The case of $p \in\left(1+\frac{2 \alpha}{N}, \frac{N}{N-2 \alpha}\right) \ldots \ldots \ldots \ldots \ldots$

4.4 Proof of Theorem $1.2($ ii $)$ and $($ iii $) \ldots \ldots \ldots \ldots$

Key words: Fractional Laplacian, Dirac measure, Isolated singularity, Weak solution, Weakly singular solution, Strongly singular solution.

MSC2010: 35R11, 35J75, 35R06

\footnotetext{
${ }^{1}$ chenhuyuan@yeah.net

${ }^{2}$ Laurent.Veron@lmpt.univ-tours.fr
} 


\section{Introduction}

Let $\Omega$ be a bounded $C^{2}$ domain of $\mathbb{R}^{N}(N \geq 2)$ containing $0, \alpha \in(0,1)$ and let $\delta_{0}$ denote the Dirac measure at 0 . In this paper, we study the properties of the weak solution to problem

$$
\begin{aligned}
& (-\Delta)^{\alpha} u+u^{p}=k \delta_{0} \quad \text { in } \quad \Omega \\
& u=0 \quad \text { in } \Omega^{\mathrm{c}} \text {, }
\end{aligned}
$$

where $k>0$ and $p \in\left(0, \frac{N}{N-2 \alpha}\right)$ and $(-\Delta)^{\alpha}$ is the $\alpha$-fractional Laplacian defined by

$$
(-\Delta)^{\alpha} u(x)=\lim _{\epsilon \rightarrow 0^{+}}(-\Delta)_{\epsilon}^{\alpha} u(x),
$$

where for $\epsilon>0$,

$$
(-\Delta)_{\epsilon}^{\alpha} u(x)=-\int_{\mathbb{R}^{N}} \frac{u(z)-u(x)}{|z-x|^{N+2 \alpha}} \chi_{\epsilon}(|x-z|) d z
$$

and

$$
\chi_{\epsilon}(t)= \begin{cases}0, & \text { if } \quad \mathrm{t} \in[0, \epsilon] \\ 1, & \text { if } \mathrm{t}>\epsilon .\end{cases}
$$

In 1980, Benilan and Brezis (see $[2,1]$ ) studied the case $\alpha=1$ in equation (1.1) and proved in particular that equation

$$
\begin{aligned}
& -\Delta u+u^{q}=k \delta_{0} \quad \text { in } \quad \Omega \\
& u=0 \quad \text { on } \quad \partial \Omega
\end{aligned}
$$

admits a unique solution $u_{k}$ for $1<q<N /(N-2)$, while no solution exists when $q \geq N /(N-2)$. Soon after, Brezis and Véron [3] proved that the problem

$$
\begin{array}{rlll}
-\Delta u+u^{q}=0 & \text { in } & \Omega \backslash\{0\} \\
u=0 & \text { on } & \partial \Omega
\end{array}
$$

admits only the zero solution when $q \geq N /(N-2)$. When $1<q<N /(N-$ $2)$, Véron in [13] obtained the description of the all the possible singular behaviour of the positive solutions of (1.3). In particular he proved that this behaviour is always isotropic (when $(N+1) /(N-1) \leq q<N /(N-2)$ the assumption of positivity is unnecessary) and that two types of singular behaviour occur:

(i) either $u(x) \sim c_{N} k|x|^{2-N}$ when $x \rightarrow 0$ and $k$ can take any positive value; $u$ is said to have a weak singularity at 0 , and actually $u=u_{k}$.

(ii) or $u(x) \sim c_{N, q}|x|^{-\frac{2}{q-1}}$ when $x \rightarrow 0$ and $u$ has a strong singularity at 0 , and $u=u_{\infty}:=\lim _{k \rightarrow \infty} u_{k}$.

A large series of papers has been devoted to the extension of semilinear problems involving the Laplacian to problems where the diffusion operator 
is non-local, the most classical one being the fractional Laplacian, see e.g. $[4,5,9,10,11]$. In a recent work, Chen and Véron [7] considered the problem

$$
\begin{array}{rll}
(-\Delta)^{\alpha} u+u^{p}=0 & \text { in } \quad \Omega \backslash\{0\} \\
u=0 & \text { in } \quad \Omega^{\mathrm{c}},
\end{array}
$$

where $1+\frac{2 \alpha}{N}<p<p_{\alpha}^{*}:=\frac{N}{N-2 \alpha}$. They proved that (1.4) admits a singular solution $u_{s}$ which satisfies

$$
\lim _{x \rightarrow 0} u_{s}(x)|x|^{\frac{2 \alpha}{p-1}}=c_{0},
$$

for some $c_{0}>0$. Moreover $u_{s}$ is the unique positive solution of (1.4) such that

$$
0<\liminf _{x \rightarrow 0} u(x)|x|^{\frac{2 \alpha}{p-1}} \leq \limsup _{x \rightarrow 0} u(x)|x|^{\frac{2 \alpha}{p-1}}<\infty .
$$

In this article we will call weakly singular solution a solution $u$ of (1.4) which satisfies $\lim _{\sup _{x \rightarrow 0}}|u(x)||x|^{N-2 \alpha}<\infty$ and strongly singular solution if $\lim _{x \rightarrow 0}|u(x)||x|^{N-2 \alpha}=\infty$.

The existence of solutions of (1.1) is a particular case of the more general problem

$$
\begin{aligned}
(-\Delta)^{\alpha} u+g(u)=\nu & \text { in } \quad \Omega \\
u=0 & \text { in } \quad \Omega^{\mathrm{c}}
\end{aligned}
$$

which has been study by Chen and Véron in [8] under the assumption that $g$ is a subcritical nonlinearity, $\nu$ being a positive and bounded Radon measure in $\Omega$.

Definition 1.1 A function u belonging to $L^{1}(\Omega)$ is a weak solution of (1.7) if $g(u) \in L^{1}\left(\Omega, \rho^{\alpha} d x\right)$ and

$$
\int_{\Omega}\left[u(-\Delta)^{\alpha} \xi+g(u) \xi\right] d x=\int_{\Omega} \xi d \nu \quad \forall \xi \in \mathbb{X}_{\alpha},
$$

where $\rho(x):=\operatorname{dist}\left(x, \Omega^{c}\right)$ and $\mathbb{X}_{\alpha} \subset C\left(\mathbb{R}^{N}\right)$ is the space of functions $\xi$ satisfying:

(i) $\operatorname{supp}(\xi) \subset \bar{\Omega}$,

(ii) $(-\Delta)^{\alpha} \xi(x)$ exists for all $x \in \Omega$ and $\left|(-\Delta)^{\alpha} \xi(x)\right| \leq c_{1}$ for some $c_{1}>0$,

(iii) there exist $\varphi \in L^{1}\left(\Omega, \rho^{\alpha} d x\right)$ and $\epsilon_{0}>0$ such that $\left|(-\Delta)_{\epsilon}^{\alpha} \xi\right| \leq \varphi$ a.e. in $\Omega$, for all $\epsilon \in\left(0, \epsilon_{0}\right]$.

According to Theorem 1.1 in [8], problem (1.1) admits a unique weak solution $u_{k}$, moreover,

$$
\mathbb{G}_{\alpha}\left[k \delta_{0}\right]-\mathbb{G}_{\alpha}\left[\left(\mathbb{G}_{\alpha}\left[k \delta_{0}\right]\right)^{p}\right] \leq u_{k} \leq \mathbb{G}_{\alpha}\left[k \delta_{0}\right] \text { in } \Omega,
$$


where $\mathbb{G}_{\alpha}[\cdot]$ is the Green operator defined by

$$
\mathbb{G}_{\alpha}[\nu](x)=\int_{\Omega} G_{\alpha}(x, y) d \nu(y), \quad \forall \nu \in \mathfrak{M}\left(\Omega, \rho^{\alpha}\right),
$$

with $G_{\alpha}$ is the Green kernel of $(-\Delta)^{\alpha}$ in $\Omega$ and $\mathfrak{M}\left(\Omega, \rho^{\alpha}\right)$ denotes the space of Radon measures in $\Omega$ such that $\int_{\Omega} \rho^{\alpha} d|\nu|<\infty$. By (1.9),

$$
\lim _{x \rightarrow 0} u_{k}(x)|x|^{N-2 \alpha}=c_{\alpha, N} k .
$$

for some $c_{\alpha, N}>0$. From Theorem 1.1 in [8], there holds

$$
u_{k}(x) \leq u_{k+1}(x), \quad \forall x \in \Omega
$$

then there exists

$$
u_{\infty}(x)=\lim _{k \rightarrow \infty} u_{k}(x) \quad \forall x \in \mathbb{R}^{N} \backslash\{0\},
$$

and $u_{\infty}(x) \in \mathbb{R}_{+} \cup\{+\infty\}$.

Motivated by these results and in view of the nonlocal character of the fractional Laplacian, in this article we analyse the connection between the solutions of (1.1) and the ones of (1.4). Our main result is the following

Theorem 1.1 Assume that $1+\frac{2 \alpha}{N} \geq \frac{2 \alpha}{N-2 \alpha}$ and $p \in\left(0, p_{\alpha}^{*}\right)$. Then $u_{k}$ is a classical solution of (1.4). Furthermore,

(i) if $p \in\left(0,1+\frac{2 \alpha}{N}\right)$,

$$
u_{\infty}(x)=\infty \quad \forall x \in \Omega
$$

(ii) if $p \in\left(1+\frac{2 \alpha}{N}, p_{\alpha}^{*}\right)$,

$$
u_{\infty}=u_{s}
$$

where $u_{s}$ is the solution of (1.4) satisfying (1.5).

Moreover, if $1+\frac{2 \alpha}{N}=\frac{2 \alpha}{N-2 \alpha}$, (1.14) holds for $p=1+\frac{2 \alpha}{N}$.

The result of part $(i)$ indicates that even if the absorption is superlinear, the diffusion dominates and there is no strongly singular solution to problem (1.4). On the contrary, part (ii) points out that the absorption dominates the diffusion; the limit function $u_{s}$ is the least strongly singular solution of (1.4). Comparing Theorem 1.1 with the results for Laplacian case, part $(i)$ with $p \in(0,1]$ and $(i i)$ are similar as the Laplacian case, but part $(i)$ with $p \in\left(1,1+\frac{2 \alpha}{N}\right]$ is totally different from the one in the case $\alpha=1$. This striking phenomenon comes comes from the fact that the fractional Laplacian is a nonlocal operator, which requires the solution to belong to $L^{1}(\Omega)$, therefore no local barrier can be constructed if $p$ is too close to 1 .

At end, we consider the case where $1+\frac{2 \alpha}{N}<\frac{2 \alpha}{N-2 \alpha}$. It occurs when $N=2$ and $\frac{\sqrt{5}-1}{2}<\alpha<1$ or $N=3$ and $\frac{3(\sqrt{5}-1)}{4}<\alpha<1$. In this situation, we have the following results. 
Theorem 1.2 Assume that $1+\frac{2 \alpha}{N}<\frac{2 \alpha}{N-2 \alpha}$ and $p \in\left(0, p_{\alpha}^{*}\right)$. Then $u_{k}$ is a classical solution of (1.4). Furthermore,

(i) if $p \in\left(0, \frac{N}{2 \alpha}\right)$, then

$$
u_{\infty}(x)=\infty \quad \forall x \in \Omega
$$

(ii) if $p \in\left(1+\frac{2 \alpha}{N}, \frac{2 \alpha}{N-2 \alpha}\right)$, then $u_{\infty}$ is a classical solution of (1.4) and there exist $\rho_{0}>0$ and $c_{2}>0$ such that

$$
c_{2}|x|^{-\frac{(N-2 \alpha) p}{p-1}} \leq u_{\infty} \leq u_{s} \quad \forall x \in B_{\rho_{0}} \backslash\{0\} ;
$$

(iii) if $p=\frac{2 \alpha}{N-2 \alpha}$, then $u_{\infty}$ is a classical solution of (1.4) and there exist $\rho_{0}>0$ and $c_{3}>0$ such that

$$
c_{3} \frac{|x|^{-\frac{(N-2 \alpha) p}{p-1}}}{(1+|\log (|x|)|)^{\frac{1}{p-1}}} \leq u_{\infty} \leq u_{s} \quad \forall x \in B_{\rho_{0}} \backslash\{0\} ;
$$

(iv) if $p \in\left(\frac{2 \alpha}{N-2 \alpha}, p_{\alpha}^{*}\right)$, then

$$
u_{\infty}=u_{s}
$$

We remark that $\frac{N}{2 \alpha}<1+\frac{2 \alpha}{N}$ if $1+\frac{2 \alpha}{N}<\frac{2 \alpha}{N-2 \alpha}$. Therefore Theorem 1.2 does not provide any description of $u_{\infty}$ in the region

$$
\mathcal{U}:=\left\{(\alpha, p) \in(0,1) \times\left(1, \frac{N}{N-2}\right): \frac{N}{2 \alpha}<1+\frac{2 \alpha}{N}, \frac{N}{2 \alpha}<p<1+\frac{2 \alpha}{N}\right\} .
$$

Furthermore, in parts $(i i)$ and $(i i i)$, we do not obtain that $u_{\infty}=u_{s}$, since (1.15) and (1.16) do not provide sharp estimates on $u_{\infty}$ in order it to belong to the uniqueness class characterized by (1.6).

The paper is organized as follows. In Section 2, we present some some estimates for the Green kernel and comparison principles. In Section 3, we prove that the weak solution of (1.1) is a classical solution of (1.4). Section 4 is devoted to analyze the limit of weakly singular solutions as $k \rightarrow \infty$.

\section{Preliminaries}

The purpose of this section is to recall some known results. We denote by $B_{r}(x)$ the ball centered at $x$ with radius $r$ and $B_{r}:=B_{r}(0)$.

Lemma 2.1 Assume that $0<p<p_{\alpha}^{*}$, then there exists $c_{4}, c_{5}, c_{6}>1$ such that

(i) if $p \in\left(0, \frac{2 \alpha}{N-2 \alpha}\right)$,

$$
\frac{1}{c_{4}} \leq \mathbb{G}_{\alpha}\left[\left(\mathbb{G}_{\alpha}\left[\delta_{0}\right]\right)^{p}\right] \leq c_{4} \quad \text { in } \quad B_{r} \backslash\{0\}
$$


(ii) if $p=\frac{2 \alpha}{N-2 \alpha}$,

$$
-\frac{1}{c_{5}} \ln |x| \leq \mathbb{G}_{\alpha}\left[\left(\mathbb{G}_{\alpha}\left[\delta_{0}\right]\right)^{p}\right] \leq-c_{5} \ln |x| \quad \text { in } \quad B_{r} \backslash\{0\} ;
$$

(iii) if $p \in\left(\frac{2 \alpha}{N-2 \alpha}, p_{\alpha}^{*}\right)$,

$$
\frac{1}{c_{6}}|x|^{2 \alpha-(N-2 \alpha) p} \leq \mathbb{G}_{\alpha}\left[\left(\mathbb{G}_{\alpha}\left[\delta_{0}\right]\right)^{p}\right] \leq c_{6}|x|^{2 \alpha-(N-2 \alpha) p} \quad \text { in } \quad B_{r} \backslash\{0\},
$$

where $r=\frac{1}{4} \min \{1, \operatorname{dist}(0, \partial \Omega)\}$ and $\mathbb{G}_{\alpha}$ is defined by (1.9).

Proof. The proof follows easily from Chen-Song's estimates of Green functions [9], see [6, Theorem 5.2] for a detailled computation.

Theorem 2.1 Assume that $O$ is a bounded domain of $\mathbb{R}^{N}$ and $u_{1}, u_{2}$ are continuous in $\bar{O}$ and satisfy

$$
(-\Delta)^{\alpha} u+u^{p}=0 \quad \text { in } \quad O .
$$

Moreover, we assume that $u_{1} \geq u_{2}$ in $O^{c}$. Then,

(i) either $u_{1}>u_{2}$ in $O$,

(ii) or $u_{1} \equiv u_{2} \quad$ a.e. in $\mathbb{R}^{N}$.

Proof. The proof refers to [5, Theorem 2.3] (see also [4, Theorem 5.2]).

The following stability result is proved in [5, Theorem 2.2].

Theorem 2.2 Suppose that $\mathcal{O}$ is a bounded $C^{2}$ domain and $h: \mathbb{R} \rightarrow \mathbb{R}$ is continuous. Assume $\left\{u_{n}\right\}$ is a sequence of functions, uniformly bounded in $L^{1}\left(\mathcal{O}^{c}, \frac{d y}{1+|y|^{N+2 \alpha}}\right)$, satisfying

$$
(-\Delta)^{\alpha} u_{n}+h\left(u_{n}\right) \geq f_{n}\left(\operatorname{resp}(-\Delta)^{\alpha} u_{n}+h\left(u_{n}\right) \leq f_{n}\right) \quad \text { in } \mathcal{O}
$$

in the viscosity sense, where the $f_{n}$ are continuous in $\mathcal{O}$. If there holds

(i) $u_{n} \rightarrow u$ locally uniformly in $\mathcal{O}$,

(ii) $u_{n} \rightarrow u$ in $L^{1}\left(\mathbb{R}^{N}, \frac{d y}{1+|y|^{N+2 \alpha}}\right)$,

(iii) $f_{n} \rightarrow f$ locally uniformly in $\mathcal{O}$,

then

$$
(-\Delta)^{\alpha} u+h(u) \geq f\left(\operatorname{resp}(-\Delta)^{\alpha} u+h(u) \leq f\right) \quad \text { in } \mathcal{O}
$$

in the viscosity sense. 


\section{Regularity}

In this section, we prove that any weak solution of (1.1) is a classical solution of (1.4). To this end, we introduce some auxiliary lemma.

Lemma 3.1 Assume that $w \in C^{2 \alpha+\epsilon}\left(\bar{B}_{1}\right)$ with $\epsilon>0$ satisfies

$$
(-\Delta)^{\alpha} w=h \quad \text { in } \quad B_{1}
$$

where $h \in C^{1}\left(\bar{B}_{1}\right)$. Then for $\beta \in(0,2 \alpha)$, there exists $c_{7}>0$ such that

$$
\|w\|_{C^{\beta}\left(\bar{B}_{1 / 4}\right)} \leq c_{7}\left(\|w\|_{L^{\infty}\left(B_{1}\right)}+\|h\|_{L^{\infty}\left(B_{1}\right)}+\left\|(1+|\cdot|)^{-N-2 \alpha} w\right\|_{L^{1}\left(\mathbb{R}^{N}\right)}\right) .
$$

Proof. Let $\eta: \mathbb{R}^{N} \rightarrow[0,1]$ be a $C^{\infty}$ function such that

$$
\eta=1 \quad \text { in } \quad B_{\frac{3}{4}} \text { and } \eta=0 \text { in } B_{1}^{c} .
$$

We denote $v=w \eta$, then $v \in C^{2 \alpha+\epsilon}\left(\mathbb{R}^{N}\right)$ and for $x \in B_{\frac{1}{2}}, \epsilon \in\left(0, \frac{1}{4}\right)$,

$$
\begin{aligned}
(-\Delta)_{\epsilon}^{\alpha} v(x) & =-\int_{\mathbb{R}^{N} \backslash B_{\epsilon}} \frac{v(x+y)-v(x)}{|y|^{N+2 \alpha}} d y \\
& =(-\Delta)_{\epsilon}^{\alpha} w(x)+\int_{\mathbb{R}^{N} \backslash B_{\epsilon}} \frac{(1-\eta(x+y)) w(x+y)}{|y|^{N+2 \alpha}} d y .
\end{aligned}
$$

Together with the fact of $\eta(x+y)=1$ for $y \in B_{\epsilon}$, we have

$\int_{\mathbb{R}^{N} \backslash B_{\epsilon}} \frac{(1-\eta(x+y)) w(x+y)}{|y|^{N+2 \alpha}} d y=\int_{\mathbb{R}^{N}} \frac{(1-\eta(x+y)) w(x+y)}{|y|^{N+2 \alpha}} d y=: h_{1}(x)$,

thus,

$$
(-\Delta)^{\alpha} v=h+h_{1} \quad \text { in } \quad B_{\frac{1}{2}} .
$$

For $x \in B_{\frac{1}{2}}$ and $z \in \mathbb{R}^{N} \backslash B_{\frac{3}{4}}$, there holds

$$
|z-x| \geq|z|-|x| \geq|z|-\frac{1}{2} \geq \frac{1}{16}(1+|z|)
$$

which implies

$$
\begin{aligned}
\left|h_{1}(x)\right|=\left|\int_{\mathbb{R}^{N}} \frac{(1-\eta(z)) w(z)}{|z-x|^{N+2 \alpha}} d z\right| & \leq \int_{\mathbb{R}^{N} \backslash B_{\frac{3}{4}}} \frac{|w(z)|}{|z-x|^{N+2 \alpha}} d z \\
& \leq 16^{N+2 \alpha} \int_{\mathbb{R}^{N}} \frac{|w(z)|}{(1+|z|)^{N+2 \alpha}} d z \\
& =16^{N+2 \alpha}\left\|(1+|\cdot|)^{-N-2 \alpha} w\right\|_{L^{1}\left(\mathbb{R}^{N}\right)} .
\end{aligned}
$$


By [11, Proposition 2.1.9], for $\beta \in(0,2 \alpha)$, there exists $c_{8}>0$ such that

$$
\begin{aligned}
\|v\|_{C^{\beta}\left(\bar{B}_{1 / 4}\right)} & \leq c_{8}\left(\|v\|_{L^{\infty}\left(\mathbb{R}^{N}\right)}+\left\|h+h_{1}\right\|_{L^{\infty}\left(B_{1 / 2}\right)}\right) \\
& \leq c_{8}\left(\|w\|_{L^{\infty}\left(B_{1}\right)}+\|h\|_{L^{\infty}\left(B_{1}\right)}+\left\|h_{1}\right\|_{L^{\infty}\left(B_{1 / 2}\right)}\right) \\
& \leq c_{9}\left(\|w\|_{L^{\infty}\left(B_{1}\right)}+\|h\|_{L^{\infty}\left(B_{1}\right)}+\left\|(1+|\cdot|)^{-N-2 \alpha} w\right\|_{L^{1}\left(\mathbb{R}^{N}\right)}\right)
\end{aligned}
$$

where $c_{9}=16^{N+2 \alpha} c_{8}$. Combining with $w=v$ in $B_{\frac{3}{4}}$, we obtain (3.1).

Theorem 3.1 Let $\alpha \in(0,1)$ and $0<p<p_{\alpha}^{*}$, then the weak solution of (1.1) is a classical solution of (1.4).

Proof. Let $u_{k}$ be the weak solution of (1.1). By [8, Theorem 1.1], we have

$$
0 \leq u_{k}=\mathbb{G}_{\alpha}\left[k \delta_{0}\right]-\mathbb{G}_{\alpha}\left[u_{k}^{p}\right] \leq \mathbb{G}_{\alpha}\left[k \delta_{0}\right] .
$$

We observe that $\mathbb{G}_{\alpha}\left[k \delta_{0}\right]=k \mathbb{G}_{\alpha}\left[\delta_{0}\right]=k G_{\alpha}(\cdot, 0)$ is $C_{\text {loc }}^{2}(\Omega \backslash\{0\})$. Denote by $O$ an open set satisfying $\bar{O} \subset \Omega \backslash B_{r}$ with $r>0$. Then $\mathbb{G}_{\alpha}\left[k \delta_{0}\right]$ is uniformly bounded in $\Omega \backslash B_{r / 2}$, so is $u_{k}^{p}$ by (3.2).

Let $\left\{g_{n}\right\}$ be a sequence nonnegative functions in $C_{0}^{\infty}\left(\mathbb{R}^{N}\right)$ such that $g_{n} \rightarrow \delta_{0}$ in the weak sense of measures and let $w_{n}$ be the solution of

$$
\begin{aligned}
(-\Delta)^{\alpha} u+u^{p} & =k g_{n} & & \text { in } \quad \\
u & =0 & & \text { in } \quad \Omega^{\mathrm{c}} .
\end{aligned}
$$

From [8], we obtain that

$$
u_{k}=\lim _{n \rightarrow \infty} w_{n} \quad \text { a.e. in } \Omega .
$$

We observe that $0 \leq w_{n}=\mathbb{G}_{\alpha}\left[k g_{n}\right]-\mathbb{G}_{\alpha}\left[w_{n}^{p}\right] \leq k \mathbb{G}_{\alpha}\left[g_{n}\right]$ and $\mathbb{G}_{\alpha}\left[g_{n}\right]$ converges to $\mathbb{G}_{\alpha}\left[\delta_{0}\right]$ uniformly in any compact set of $\Omega \backslash\{0\}$ and in $L^{1}(\Omega)$; then there exists $c_{10}>0$ independent of $n$ such that

$$
\left\|w_{n}\right\|_{L^{\infty}\left(\Omega \backslash B_{r / 2}\right)} \leq c_{10} k \text { and }\left\|w_{n}\right\|_{L^{1}(\Omega)} \leq c_{10} k .
$$

By [10, Corollary 2.4] and Lemma 3.1, there exist $\epsilon>0, \beta \in(0,2 \alpha)$ and positive constants $c_{11}, c_{12}, c_{13}>0$ independent of $n$ and $k$, such that

$$
\begin{aligned}
& \left\|w_{n}\right\|_{C^{2 \alpha+\epsilon}(O)} \leq c_{11}\left(\left\|w_{n}\right\|_{L^{\infty}\left(\Omega \backslash B_{\frac{r}{2}}\right)}^{p}+\left\|k g_{n}\right\|_{L^{\infty}\left(\Omega \backslash B_{\frac{r}{2}}\right)}+\left\|w_{n}\right\|_{C^{\beta}\left(\Omega \backslash B_{\frac{3 r}{2}}\right)}\right) \\
& \quad \leq c_{12}\left(\left\|w_{n}\right\|_{L^{\infty}\left(\Omega \backslash B_{\frac{r}{2}}\right)}^{p}+\left\|w_{n}\right\|_{L^{\infty}\left(\Omega \backslash B_{\frac{r}{2}}\right)}+\left\|k g_{n}\right\|_{L^{\infty}\left(\Omega \backslash B_{\frac{r}{2}}\right)}+\left\|w_{n}\right\|_{L^{1}(\Omega)}\right) \\
& \quad \leq c_{13}\left(k+k^{p}\right) .
\end{aligned}
$$

Therefore, together with (3.4) and the Arzela-Ascoli Theorem, it follows that $u_{k} \in C^{2 \alpha+\frac{\epsilon}{2}}(O)$. This implies that $u_{k}$ is $C^{2 \alpha+\frac{\epsilon}{2}}$ locally in $\Omega \backslash\{0\}$. Therefore, $w_{n} \rightarrow u_{k}$ and $g_{n} \rightarrow 0$ uniformly in any compact subset of $\Omega \backslash\{0\}$ as $n \rightarrow \infty$. We conclude that $u_{k}$ is a classical solution of (1.4) by Theorem 2.2 . 
Corollary 3.1 Let $u_{k}$ be the weak solution of (1.1) and $O$ be an open set satisfying $\bar{O} \subset \Omega \backslash B_{r}$ with $r>0$. Then there exist $\epsilon>0$ and $c_{14}>0$ independent of $k$ such that

$$
\left\|u_{k}\right\|_{C^{2 \alpha+\epsilon}(O)} \leq c_{14}\left(\left\|u_{k}\right\|_{L^{\infty}\left(\Omega \backslash B_{\frac{r}{2}}\right)}^{p}+\left\|u_{k}\right\|_{L^{\infty}\left(\Omega \backslash B_{\frac{r}{2}}\right)}+\left\|u_{k}\right\|_{L^{1}(\Omega)}\right) .
$$

Proof. By Theorem 3.1, $u_{k}$ is a solution of (1.4). Then the result follows from [10, Corollary 2.4] and Lemma 3.1 since there exist $\epsilon>0, \beta \in(0,2 \alpha)$ and constants $c_{15}, c_{16}>0$, independent of $k$, such that

$$
\begin{aligned}
\left\|u_{k}\right\|_{C^{2 \alpha+\epsilon}(O)} & \leq c_{15}\left(\left\|u_{k}\right\|_{L^{\infty}\left(\Omega \backslash B_{\frac{r}{2}}\right)}^{p}+\left\|u_{k}\right\|_{C^{\beta}\left(\Omega \backslash B_{\frac{3 r}{4}}\right)}\right) \\
& \leq c_{16}\left(\left\|u_{k}\right\|_{L^{\infty}\left(\Omega \backslash B_{\frac{r}{2}}\right)}^{p}+\left\|u_{k}\right\|_{L^{\infty}\left(\Omega \backslash B_{\frac{r}{2}}\right)}+\left\|u_{k}\right\|_{L^{1}(\Omega)}\right) .
\end{aligned}
$$

Theorem 3.2 Assume that the weak solutions $u_{k}$ of (1.1) satisfy

$$
\left\|u_{k}\right\|_{L^{1}(\Omega)} \leq c_{17}
$$

for some $c_{17}>0$ independent of $k$ and that for any $r \in(0, \operatorname{dist}(0, \partial \Omega))$, there exists $c_{18}>0$ independent of $k$ such that

$$
\left\|u_{k}\right\|_{L^{\infty}\left(\Omega \backslash B_{\frac{r}{2}}\right)} \leq c_{18}
$$

Then $u_{\infty}$ is a classical solution of (1.4).

Proof. Let $O$ be an open set satisfying $\bar{O} \subset \Omega \backslash B_{r}$ for $0<r<\operatorname{dist}(0, \partial \Omega)$. By (3.5), (3.6) and (3.7), there exist $\epsilon>0$ and $c_{19}>0$ independent of $k$ such that

$$
\left\|u_{k}\right\|_{C^{2 \alpha+\epsilon}(O)} \leq c_{19} .
$$

Together with (1.13) and the Arzela-Ascoli Theorem, it implies that $u_{\infty}$ belongs to $C^{2 \alpha+\frac{\epsilon}{2}}(O)$. Hence $u_{\infty}$ is $C^{2 \alpha+\frac{\epsilon}{2}}$, locally in $\Omega \backslash\{0\}$. Therefore, $w_{n} \rightarrow u_{k}$ and $g_{n} \rightarrow 0$ uniformly in any compact set of $\Omega \backslash\{0\}$ as $n \rightarrow \infty$. Applying Theorem 2.2 we conclude that $u_{\infty}$ is a classical solution of (1.4).

\section{The limit of weakly singular solutions}

We recall that $u_{k}$ denotes the weak solution of $(1.1)$ and $d=\min \{1, \operatorname{dist}(0, \partial \Omega)\}$. 


\subsection{The case $p \in\left(0,1+\frac{2 \alpha}{N}\right]$}

Proposition 4.1 Let $p \in(0,1]$, then $\lim _{k \rightarrow \infty} u_{k}(x)=\infty$ for $x \in \Omega$.

Proof. We observe that $\mathbb{G}_{\alpha}\left[\delta_{0}\right], \mathbb{G}_{\alpha}\left[\left(\mathbb{G}_{\alpha}\left[\delta_{0}\right]\right)^{p}\right]>0$ in $\Omega$. Since by (1.9)

$$
u_{k} \geq k \mathbb{G}_{\alpha}\left[\delta_{0}\right]-k^{p} \mathbb{G}_{\alpha}\left[\left(\mathbb{G}_{\alpha}\left[\delta_{0}\right]\right)^{p}\right],
$$

this implies the claim when $p \in(0,1)$, for any $x \in \Omega$. For $p=1, u_{k}=k u_{1}$. The proof follows since $u_{1}>0$ in $\Omega$.

Now we consider the case of $p \in\left(1,1+\frac{2 \alpha}{N}\right]$. Let $\left\{r_{k}\right\} \subset\left(0, \frac{d}{2}\right]$ be a strictly decreasing sequence of numbers satisfying $\lim _{k \rightarrow \infty} r_{k}=0$. Denote by $\left\{z_{k}\right\}$ the sequence of functions defined by

$$
z_{k}(x)= \begin{cases}-d^{-N}, & x \in B_{r_{k}} \\ |x|^{-N}-d^{-N}, & x \in B_{r_{k}}^{c} .\end{cases}
$$

Lemma 4.1 Let $\left\{\rho_{k}\right\}$ be a strictly decreasing sequence of numbers such that $\frac{r_{k}}{\rho_{k}}<\frac{1}{2}$ and $\lim _{k \rightarrow \infty} \frac{r_{k}}{\rho_{k}}=0$. Then

$$
(-\Delta)^{\alpha} z_{k}(x) \leq-c_{1, k}|x|^{-N-2 \alpha}, \quad x \in B_{\rho_{k}}^{c}
$$

where $c_{1, k}=-c_{20} \log \left(\frac{r_{k}}{\rho_{k}}\right)$ with $c_{20}>0$ independent of $k$.

Proof. For any $x \in B_{\rho_{k}}^{c}$, there holds

$$
\begin{aligned}
& (-\Delta)^{\alpha} z_{k}(x)=-\frac{1}{2} \int_{\mathbb{R}^{N}} \frac{z_{k}(x+y)+z_{k}(x-y)-2 z_{k}(x)}{|y|^{N+2 \alpha}} d y \\
& =-\frac{1}{2} \int_{\mathbb{R}^{N}} \frac{|x+y|^{-N} \chi_{B_{r_{k}}^{c}(-x)}(y)+|x-y|^{-N} \chi_{B_{r_{k}}^{c}(x)}(y)-2|x|^{-N}}{|y|^{N+2 \alpha}} d y \\
& =-\frac{1}{2}|x|^{-N-2 \alpha} \int_{\mathbb{R}^{N}} \frac{\delta\left(x, z, r_{k}\right)}{|z|^{N+2 \alpha}} d z
\end{aligned}
$$

where $\delta\left(x, z, r_{k}\right)=\left|z+e_{x}\right|^{-N} \chi_{B_{\frac{r_{k}}{|x|}}^{c}\left(-e_{x}\right)}(z)+\left|z-e_{x}\right|^{-N} \chi_{B_{\frac{r_{k}}{|x|}}^{c}\left(e_{x}\right)}(z)-2$ and $e_{x}=\frac{x}{|x|}$.

We observe that $\frac{r_{k}}{|x|} \leq \frac{r_{k}}{\rho_{k}}<\frac{1}{2}$ and $\left|z \pm e_{x}\right| \geq 1-|z| \geq \frac{1}{2}$ for $z \in B_{\frac{1}{2}}$. Then there exists $c_{21}>0$ such that

$$
\left|\delta\left(x, z, r_{k}\right)\right|=|| z+\left.e_{x}\right|^{-N}+\left|z-e_{x}\right|^{-N}-\left.2\left|\leq c_{21}\right| z\right|^{2} .
$$

Therefore,

$$
\begin{aligned}
\left|\int_{B_{\frac{1}{2}}(0)} \frac{\delta\left(x, z, r_{k}\right)}{|z|^{N+2 \alpha}} d z\right| & \leq \int_{B_{\frac{1}{2}}(0)} \frac{\left|\delta\left(x, z, r_{k}\right)\right|}{|z|^{N+2 \alpha}} d z \\
& \leq c_{21} \int_{B_{\frac{1}{2}}(0)}|z|^{2-N-2 \alpha} d z \leq c_{22}
\end{aligned}
$$


where $c_{22}>0$ is independent of $k$.

When $z \in B_{\frac{1}{2}}\left(-e_{x}\right)$ there holds

$$
\begin{aligned}
\int_{B_{\frac{1}{2}}\left(-e_{x}\right)} \frac{\delta\left(x, z, r_{k}\right)}{|z|^{N+2 \alpha}} d z & \geq \int_{B_{\frac{1}{2}}^{c}\left(-e_{x}\right)} \frac{\left|z+e_{x}\right|^{-N} \chi_{B_{\frac{r_{k}}{|x|}}\left(-e_{x}\right)}(z)-2}{|z|^{N+2 \alpha}} d z \\
& \geq c_{23} \int_{B_{\frac{1}{2}}(0) \backslash B_{\frac{r_{k}}{|x|}(0)}\left(|z|^{-N}-2\right) d z} \\
& \geq-c_{24} \log \left(\frac{r_{k}}{|x|}\right) \geq-c_{24} \log \left(\frac{r_{k}}{\rho_{k}}\right),
\end{aligned}
$$

where $c_{23}, c_{24}>0$ are independent of $k$.

For $z \in B_{\frac{1}{2}}\left(e_{x}\right)$, we have

$$
\int_{B_{\frac{1}{2}}\left(e_{x}\right)} \frac{\delta\left(x, z, r_{k}\right)}{|z|^{N+2 \alpha}} d z=\int_{B_{\frac{1}{2}}\left(-e_{x}\right)} \frac{\delta\left(x, z, r_{k}\right)}{|z|^{N+2 \alpha}} d z .
$$

Finally, for $z \in O:=\mathbb{R}^{N} \backslash\left(B_{\frac{1}{2}}(0) \cup B_{\frac{1}{2}}\left(-e_{x}\right) \cup B_{\frac{1}{2}}\left(e_{x}\right)\right)$, we have

$$
\left|\int_{O} \frac{\delta\left(x, z, r_{k}\right)}{|z|^{N+2 \alpha}} d z\right| \leq c_{25} \int_{B_{\frac{1}{2}}^{c}(0)} \frac{|z|^{-N}+1}{|z|^{N+2 \alpha}} d z \leq c_{26}
$$

where $c_{25}, c_{26}>0$ are independent of $k$.

Combining these inequalities we obtain that there exists $c_{20}>0$ independent of $k$ such that

$$
(-\Delta)^{\alpha} z_{k}(x)|x|^{N+2 \alpha} \leq c_{20} \log \left(\frac{r_{k}}{\rho_{k}}\right):=c_{1, k},
$$

which ends the proof.

Proposition 4.2 Assume that

$$
\frac{2 \alpha}{N-2 \alpha}<1+\frac{2 \alpha}{N}, \quad \max \left\{1, \frac{2 \alpha}{N-2 \alpha}\right\}<p<1+\frac{2 \alpha}{N}
$$

and $z_{k}$ is defined by (4.1) with $r_{k}=k^{-\frac{p-1}{N-(N-2 \alpha) p}}(\log k)^{-2}$. Then there exists $k_{0}>0$ such that for any $k \geq k_{0}$

$$
u_{k} \geq c_{2, k}^{\frac{1}{p-1}} z_{k} \quad \text { in } \quad B_{d}
$$

where $c_{2, k}=\ln \ln k$. 
Proof. For $p \in\left(\max \left\{1, \frac{2 \alpha}{N-2 \alpha}\right\}, 1+\frac{2 \alpha}{N}\right)$, it follows by (1.9) and Lemma 2.1-(iii) that there exist $\rho_{0} \in(0, d)$ and $c_{27}, c_{28}>0$ independent of $k$ such that, for $x \in \bar{B}_{\rho_{0}} \backslash\{0\}$,

$$
\begin{aligned}
u_{k}(x) & \geq k \mathbb{G}_{\alpha}\left[\delta_{0}\right](x)-k^{p} \mathbb{G}_{\alpha}\left[\left(\mathbb{G}_{\alpha}\left[\delta_{0}\right]\right)^{p}\right](x) \\
& \geq c_{27} k|x|^{-N+2 \alpha}-c_{28} k^{p}|x|^{-(N-2 \alpha) p+2 \alpha} \\
& =c_{27} k|x|^{-N+2 \alpha}\left(1-\frac{c_{28}}{c_{27}} k^{p-1}|x|^{N-(N-2 \alpha) p}\right) .
\end{aligned}
$$

We choose

$$
\rho_{k}=k^{-\frac{p-1}{N-(N-2 \alpha) p}}(\log k)^{-1} .
$$

There exits $k_{1}>1$ such that for $k \geq k_{1}$

$$
\begin{aligned}
u_{k}(x) & \geq c_{27} k|x|^{-N+2 \alpha}\left(1-\frac{c_{28}}{c_{27}} k^{p-1} \rho_{k}^{N-(N-2 \alpha) p}\right) \\
& \geq \frac{c_{27}}{2} k|x|^{-N+2 \alpha}, \quad x \in \bar{B}_{\rho_{k}} \backslash\{0\} .
\end{aligned}
$$

Since $p<1+\frac{2 \alpha}{N}, 1-\frac{2 \alpha(p-1)}{N-(N-2 \alpha) p}>0$ and there exists $k_{0} \geq k_{1}$ such that

$$
\frac{c_{27}}{2} k r_{k}^{2 \alpha} \geq(\ln \ln k)^{\frac{1}{p-1}},
$$

for $k \geq k_{0}$. This implies

$$
\frac{c_{27}}{2} k|x|^{2 \alpha} \geq(\ln \ln k)^{\frac{1}{p-1}}, \quad x \in \bar{B}_{\rho_{k}} \backslash B_{r_{k}} .
$$

Together with (4.1) and (4.5), we derive

$$
u_{k}(x) \geq(\ln \ln k)^{\frac{1}{p-1}} z_{k}(x), \quad x \in \bar{B}_{\rho_{k}} \backslash B_{r_{k}},
$$

for $k \geq k_{0}$. Furthermore, it is clear that

$$
(\ln \ln k)^{\frac{1}{p-1}} z_{k}(x) \leq 0 \leq u_{k}(x)
$$

whenever $x \in B_{r_{k}}$ or $x \in B_{d}^{c}$. Set $c_{2, k}=\ln \ln k$, then by Lemma 4.1

$$
(-\Delta)^{\alpha} c_{2, k}^{\frac{1}{p-1}} z_{k}(x)+c_{2, k}^{\frac{p}{p-1}} z_{k}(x)^{p} \leq c_{2, k}^{\frac{p}{p-1}}|x|^{-N-2 \alpha}\left(-1+|x|^{N+2 \alpha-N p}\right) \leq 0,
$$

for any $x \in B_{d} \backslash B_{\rho_{k}}$, since $N+2 \alpha-N p \geq 0$ and $d \leq 1$. Applying Theorem 2.1 , we infer that

$$
c_{2, k}^{\frac{1}{p-1}} z_{k}(x) \leq u_{k}(x) \quad \forall x \in \bar{B}_{d}
$$

which ends the proof. 
Proposition 4.3 Assume

$$
1<\frac{2 \alpha}{N-2 \alpha} \leq 1+\frac{2 \alpha}{N} \text { and } p=\frac{2 \alpha}{N-2 \alpha}
$$

and let $z_{k}$ be defined by (4.1) with $r_{k}=k^{-\frac{2 \alpha}{N(N-2 \alpha)}}(\log k)^{-3}$ and $k>2$. Then there exists $k_{0}>2$ such that (4.3) holds for $k \geq k_{0}$.

Proof. By (1.9) and Lemma 2.1-(ii), there exist $\rho_{0} \in(0, d)$ and $c_{30}, c_{31}>0$ independent of $k$, such that for $x \in \bar{B}_{\rho_{0}} \backslash\{0\}$

$$
\begin{aligned}
u_{k}(x) & \geq c_{30} k|x|^{-N+2 \alpha}+c_{31} k^{p} \log |x| \\
& =c_{30} k|x|^{-N+2 \alpha}\left(1+\frac{c_{31}}{c_{30}} k^{p-1}|x|^{N-2 \alpha} \log |x|\right) .
\end{aligned}
$$

If we choose $\rho_{k}=k^{-\frac{2 \alpha}{N(N-2 \alpha)}}(\log k)^{-2}$ there exists $k_{1}>1$ such that for $k \geq k_{1}$, we have $1+\frac{c_{31}}{c_{30}} k^{p-1} \rho_{k}^{N-2 \alpha} \log \left(\rho_{k}\right) \geq \frac{1}{2}$ and

$$
u_{k}(x) \geq \frac{c_{30}}{2} k|x|^{-N+2 \alpha} \quad \forall x \in \bar{B}_{\rho_{k}} \backslash\{0\} .
$$

Since $\frac{2 \alpha}{N-2 \alpha}<1+\frac{2 \alpha}{N}$, there holds $1-\frac{4 \alpha^{2}}{N(N-2 \alpha)}>0$ and there exists $k_{0} \geq k_{1}$ such that

$$
\frac{c_{30}}{2} k r_{k}^{2 \alpha}=\frac{c_{30}}{2} k^{1-\frac{4 \alpha^{2}}{N(N-2 \alpha)}}(\log k)^{-6 \alpha} \geq(\ln \ln k)^{\frac{1}{p-1}}
$$

for $k \geq k_{0}$. The remaining of the proof is the same as in Proposition 4.2.

In the sequel, we point out the fact that the limit behavior of the $u_{k}$ depends which of the following three cases holds:

$$
\begin{aligned}
& \frac{2 \alpha}{N-2 \alpha}=1+\frac{2 \alpha}{N}=\frac{N}{2 \alpha} ; \\
& \frac{2 \alpha}{N-2 \alpha}<1+\frac{2 \alpha}{N}<\frac{N}{2 \alpha} ; \\
& \frac{2 \alpha}{N-2 \alpha}>1+\frac{2 \alpha}{N}>\frac{N}{2 \alpha} .
\end{aligned}
$$

Proposition 4.4 Assume

$$
1<\frac{2 \alpha}{N-2 \alpha} \leq 1+\frac{2 \alpha}{N} \text { and } 1<p<\frac{2 \alpha}{N-2 \alpha},
$$

or

$$
1+\frac{2 \alpha}{N}<\frac{2 \alpha}{N-2 \alpha} \text { and } 1<p<\frac{N}{2 \alpha}
$$

and $z_{k}$ is defined by (4.1) with $r_{k}=k^{-\frac{p-1}{N-2 \alpha}}(\log k)^{-1}$. Then there exists $k_{0}>2$ such that (4.3) holds for $k \geq k_{0}$. 
Proof. By (1.9) and Lemma 2.1- $(i)$, there exist $\rho_{0} \in(0, d)$ and $c_{33}, c_{34}>0$ independent of $k$ such that for $x \in \bar{B}_{\rho_{0}} \backslash\{0\}$,

$$
\begin{aligned}
u_{k}(x) & \geq c_{33} k|x|^{-N+2 \alpha}-c_{34} k^{p} \\
& =c_{33} k|x|^{-N+2 \alpha}\left(1-\frac{c_{34}}{c_{33}} k^{p-1}|x|^{N-2 \alpha}\right) .
\end{aligned}
$$

We choose $\rho_{k}=k^{-\frac{p-1}{N-2 \alpha}}$. Then there exists $k_{1}>1$ such that for $k \geq k_{1}$, $1-\frac{c_{34}}{c_{33}} k^{p-1} \rho_{k}^{N-2 \alpha} \geq \frac{1}{2}$ and

$$
u_{k}(x) \geq \frac{c_{33}}{2} k|x|^{-N+2 \alpha} \quad \forall x \in \bar{B}_{\rho_{k}} \backslash\{0\} .
$$

Clearly $p<\frac{N}{2 \alpha}$ by assumptions (4.12), (4.13), together with relations (4.9)(4.10), (4.11), thus $1-(p-1) \frac{2 \alpha}{N-2 \alpha}>0$. Therefore there exists $k_{0} \geq k_{1}$ such that

$$
\frac{c_{33}}{2} k r_{k}^{2 \alpha}=\frac{c_{33}}{2} k^{1-(p-1) \frac{2 \alpha}{N-2 \alpha}}(\log k)^{-2 \alpha} \geq(\log \log k)^{\frac{1}{p-1}}=c_{2, k}^{\frac{1}{p-1}}
$$

for $k \geq k_{0}$. The remaining of the proof is similar to the one of Proposition 4.2 .

\subsection{The case $p \in\left(1,1+\frac{2 \alpha}{N}\right]$}

We give below the proof, in two steps, of Theorem 1.1 part $(i)$ with $p \in$ $\left(1,1+\frac{2 \alpha}{N}\right]$ and Theorem 1.2 part $(i)$ with $p \in\left(1, \frac{2 \alpha}{N}\right]$.

Step 1: We claim that $u_{\infty}=\infty$ in $B_{d}$. We observe that for $\frac{2 \alpha}{N-2 \alpha}<1+\frac{2 \alpha}{N}$, Propositions 4.2, 4.3, 4.4 cover the case $p \in\left(\max \left\{1, \frac{2 \alpha}{N-2 \alpha}\right\}, 1+\frac{2 \alpha}{N}\right)$, the case $1<\frac{2 \alpha}{N-2 \alpha}<1+\frac{2 \alpha}{N}$ along with $p=\frac{2 \alpha}{N-2 \alpha}$ and the case $1<\frac{2 \alpha}{N-2 \alpha}<1+\frac{2 \alpha}{N}$ along with $p \in\left(1, \frac{2 \alpha}{N-2 \alpha}\right)$ respectively. For $\frac{2 \alpha}{N-2 \alpha}=1+\frac{2 \alpha}{N}$, Proposition 4.3, 4.4 cover the case $p=\frac{2 \alpha}{N-2 \alpha}$ and the case $p \in\left(1, \frac{2 \alpha}{N-2 \alpha}\right)$ respectively. So it covers $p \in\left(1,1+\frac{2 \alpha}{N}\right]$ in Theorem 1.1 part $(i)$. When $\frac{2 \alpha}{N-2 \alpha}>1+\frac{2 \alpha}{N}$, Proposition 4.4 covers $p \in\left(1, \frac{N}{2 \alpha}\right)$ in Theorem 1.2 part $(i)$. Therefore, we have

$$
u_{\infty} \geq c_{2, k}^{\frac{1}{p-1}} z_{k} \quad \text { in } \quad B_{d}
$$

and since for any $x \in B_{d} \backslash\{0\}, \lim _{k \rightarrow \infty} \frac{1}{c_{2, k}^{p-1}} z_{k}(x)=\infty$, we deive

$$
u_{\infty}=\infty \text { in } B_{d} .
$$

Step 2: We claim that $u_{\infty}=\infty$ in $\Omega$. By the fact of $u_{\infty}=\infty$ in $B_{d}$ and $u_{k+1} \geq u_{k}$ in $\Omega$, then for any $n>1$ there exists $k_{n}>0$ such that $u_{k_{n}} \geq n$ in $B_{d}$. For any $x_{0} \in \Omega \backslash B_{d}$, there exists $\rho>0$ such that $\bar{B}_{\rho}\left(x_{0}\right) \subset \Omega \cap B_{d / 2}^{c}$. We denote by $w_{n}$ the solution of

$$
\begin{array}{rll}
(-\Delta)^{\alpha} u+u^{p}=0 & \text { in } & B_{\rho}\left(x_{0}\right) \\
u=0 & \text { in } & B_{\rho}^{c}\left(x_{0}\right) \backslash B_{d / 2} \\
u=n & \text { in } & B_{d / 2} .
\end{array}
$$


Then by Theorem 2.1, we have

$$
u_{k_{n}} \geq w_{n}
$$

Let $\eta_{1}$ be the solution of

$$
\begin{array}{rll}
(-\Delta)^{\alpha} u=1 \quad \text { in } & B_{\rho}\left(x_{0}\right) \\
u=0 & \text { in } \quad B_{\rho}^{c}\left(x_{0}\right),
\end{array}
$$

and $v_{n}=w_{n}-n \chi_{B_{d / 2}}$, then $v_{n}=w_{n}$ in $B_{\rho}\left(x_{0}\right)$ and

$$
\begin{aligned}
(-\Delta)^{\alpha} v_{n}(x)+v_{n}^{p}(x) & =(-\Delta)^{\alpha} w_{n}(x)-n(-\Delta)^{\alpha} \chi_{B_{d / 2}}(x)+w_{n}^{p}(x) \\
& =n \int_{B_{d / 2}} \frac{d y}{|y-x|^{N+2 \alpha}} \quad \forall x \in B_{\rho}\left(x_{0}\right) .
\end{aligned}
$$

This means that $v_{n}$ is a solution of

$$
\begin{array}{rlrl}
(-\Delta)^{\alpha} u+u^{p} & =n \int_{B_{d / 2}} \frac{d y}{|y-x|^{N+2 \alpha}} & \text { in } & B_{\rho}\left(x_{0}\right), \\
u=0 & \text { in } & B_{\rho}^{c}\left(x_{0}\right) .
\end{array}
$$

It is clear that

$$
\frac{1}{c_{35}} \leq \int_{B_{d / 2}} \frac{d y}{|y-x|^{N+2 \alpha}} \leq c_{35} \quad \forall x \in B_{\rho}\left(x_{0}\right)
$$

for some $c_{35}>1$. Furhermore $\left(\frac{n}{2 c_{35} \max \eta_{1}}\right)^{\frac{1}{p}} \eta_{1}$ is sub solution of (4.17) for $n$ large enough. Then using Theorem 2.1, we obtain that

$$
v_{n} \geq\left(\frac{n}{2 c_{35} \max \eta_{1}}\right)^{\frac{1}{p}} \eta_{1} \quad \forall x \in B_{\rho}\left(x_{0}\right),
$$

which implies that

$$
w_{n} \geq\left(\frac{n}{2 c_{35} \max \eta_{1}}\right)^{\frac{1}{p}} \eta_{1} \quad \forall x \in B_{\rho}\left(x_{0}\right) .
$$

Then

$$
\lim _{n \rightarrow \infty} w_{n}\left(x_{0}\right) \rightarrow \infty
$$

Since $x_{0}$ is arbitrary and together with (4.16), it implies that $u_{\infty}=\infty$ in $\Omega$, which completes the proof.

\subsection{The case of $p \in\left(1+\frac{2 \alpha}{N}, \frac{N}{N-2 \alpha}\right)$}

Proposition 4.5 Let $\alpha \in(0,1)$ and $r_{0}=\operatorname{dist}(0, \partial \Omega)$. Then

(i) if $\max \left\{1+\frac{2 \alpha}{N}, \frac{2 \alpha}{N-2 \alpha}\right\}<p<p_{\alpha}^{*}$, there exist $R_{0} \in\left(0, r_{0}\right)$ and $c_{36}>0$ such that

$$
u_{\infty}(x) \geq c_{36}|x|^{-\frac{2 \alpha}{p-1}} \quad \forall x \in B_{R_{0}} \backslash\{0\},
$$


(ii) if $\frac{2 \alpha}{N-2 \alpha}>1+\frac{2 \alpha}{N}$ and $p=\frac{2 \alpha}{N-2 \alpha}$, there exist $R_{0} \in\left(0, r_{0}\right)$ and $c_{37}>0$ such that

$$
u_{\infty}(x) \geq \frac{c_{37}}{(1+|\log (|x|)|)^{\frac{1}{p-1}}}|x|^{-\frac{p(N-2 \alpha)}{p-1}}, \quad \forall x \in B_{R_{0}} \backslash\{0\},
$$

(iii) if $\frac{2 \alpha}{N-2 \alpha}>1+\frac{2 \alpha}{N}$ and $p \in\left(1+\frac{2 \alpha}{N}, \frac{2 \alpha}{N-2 \alpha}\right)$, there exist $R_{0} \in\left(0, r_{0}\right)$ and $c_{38}>0$ such that

$$
u_{\infty}(x) \geq c_{38}|x|^{-\frac{p(N-2 \alpha)}{p-1}} \quad \forall x \in B_{R_{0}} \backslash\{0\} .
$$

Proof. (i) Using (1.9) and Lemma 2.1(i) with $\max \left\{1+\frac{2 \alpha}{N}, \frac{2 \alpha}{N-2 \alpha}\right\}<p<p_{\alpha}^{*}$, we see that there exist $\rho_{0} \in\left(0, r_{0}\right)$ and $c_{39}, c_{40}>0$ such that

$$
u_{k}(x) \geq c_{39} k|x|^{-N+2 \alpha}-c_{40} k^{p}|x|^{-(N-2 \alpha) p+2 \alpha} \quad \forall x \in B_{\rho_{0}} \backslash\{0\} .
$$

Set

$$
\rho_{k}=\left(2^{(N-2 \alpha) p-2 \alpha-1} \frac{c_{40}}{c_{39}} k^{p-1}\right)^{\frac{1}{(N-2 \alpha)(p-1)-2 \alpha}} .
$$

Since $(N-2 \alpha)(p-1)-2 \alpha<0$, there holds $\lim _{k \rightarrow \infty} \rho_{k}=0$. Let $k_{0}>0$ such that $\rho_{k_{0}} \leq \rho_{0}$, then for $x \in B_{\rho_{k}} \backslash B_{\frac{\rho_{k}}{2}}$, we have

$$
\begin{aligned}
c_{40} k^{p}|x|^{-(N-2 \alpha) p+2 \alpha} & \leq c_{40} k^{p}\left(\frac{\rho_{k}}{2}\right)^{-(N-2 \alpha) p+2 \alpha} \\
& =\frac{c_{39}}{2} k \rho_{k}^{-N+2 \alpha} \\
& \leq \frac{c_{39}}{2} k|x|^{-N+2 \alpha}
\end{aligned}
$$

and

$$
k=\left(2^{(N-2 \alpha) p-2 \alpha-1} \frac{c_{40}}{c_{39}}\right)^{-\frac{1}{p-1}} \rho_{k}^{N-2 \alpha-\frac{2 \alpha}{p-1}} \geq c_{41}|x|^{N-2 \alpha-\frac{2 \alpha}{p-1}},
$$

where $c_{41}=\left(2^{(N-2 \alpha) p-2 \alpha-1} \frac{c_{40}}{c_{39}}\right)^{-\frac{1}{p-1}} 2^{(N-2 \alpha)(p-1)-2 \alpha-1}$. Combining with (4.18), we obtain

$$
\begin{aligned}
u_{k}(x) & =c_{39} k|x|^{-N+2 \alpha}-c_{40} k^{p}|x|^{-(N-2 \alpha) p+2 \alpha} \\
& \geq \frac{c_{39}}{2} k|x|^{-N+2 \alpha} \\
& \geq c_{42}|x|^{-\frac{2 \alpha}{p-1}}
\end{aligned}
$$

for $x \in B_{\rho_{k}} \backslash B \frac{\rho_{k}}{2}$, where $c_{42}=\frac{1}{2} c_{39} c_{41}$ is independent of $k$. By (4.22), we can choose a sequence $\left\{k_{n}\right\} \subset[1,+\infty)$ such that

$$
\rho_{k_{n+1}} \geq \frac{1}{2} \rho_{k_{n}},
$$


For any $x \in B_{\rho_{k_{0}}} \backslash\{0\}$, there exists $k_{n}$ such that $x \in B_{\rho_{k_{n}}} \backslash B_{\frac{\rho_{k_{n}}}{2}}$, then, by $(4.23)$,

$$
u_{k_{n}}(x) \geq c_{42}|x|^{-\frac{2 \alpha}{p-1}} .
$$

Together with $u_{k+1}>u_{k}$, we derive

$$
u_{\infty}(x) \geq c_{42}|x|^{-\frac{2 \alpha}{p-1}}, \quad x \in B_{\rho_{k_{0}}} \backslash\{0\} .
$$

(ii) By (1.9) and Lemma 2.1-(ii) with $p=\frac{2 \alpha}{N-2 \alpha}$, there exist $\rho_{0} \in\left(0, r_{0}\right)$ and $c_{43}, c_{44}>0$ such that

$$
u_{k}(x) \geq c_{43} k|x|^{-N+2 \alpha}-c_{44} k^{p}|\log (|x|)|, \quad x \in B_{\rho_{0}} \backslash\{0\} .
$$

Let $\left\{\rho_{k}\right\}$ be a sequence of real numbers with value in $(0,1)$ and such that

$$
c_{44} k^{p-1}\left|\log \left(\frac{\rho_{k}}{2}\right)\right|=\frac{c_{43}}{2} \rho_{k}^{-N+2 \alpha} .
$$

Then $\lim _{k \rightarrow \infty} \rho_{k}=0$ and there exists $k_{0}>0$ such that $\rho_{k_{0}} \leq \rho_{0}$. Thus, for any $x \in B_{\rho_{k}} \backslash B_{\frac{\rho_{k}}{2}}$ and $k \geq k_{0}$,

$$
c_{43} k^{p}|\log (|x|)| \leq c_{44} k^{p}\left|\log \left(\frac{\rho_{k}}{2}\right)\right|=\frac{c_{43}}{2} k \rho_{k}^{-N+2 \alpha} \leq \frac{c_{43}}{2} k|x|^{-N+2 \alpha} .
$$

Therefore, assuming always $x \in B_{\rho_{k}} \backslash B_{\frac{\rho_{k}}{2}}$, we derive from (4.25) that

$$
k=\left(\frac{c_{44}}{2 c_{43}}\right)^{-\frac{1}{p-1}}\left(\frac{\rho_{k}^{-N+2 \alpha}}{1+\left|\log \left(\rho_{k}\right)\right|}\right)^{\frac{1}{p-1}} \geq c_{45} \frac{|x|^{-\frac{N-2 \alpha}{p-1}}}{(1+|\log (|x|)|)^{\frac{1}{p-1}}},
$$

where $c_{45}=2^{-\frac{N-2 \alpha}{p-1}}\left(\frac{c_{44}}{2 c_{43}}\right)^{-\frac{1}{p-1}}$. Consequently

$$
\begin{aligned}
u_{k}(x) & \geq c_{43} k|x|^{-N+2 \alpha}-c_{44} k^{p}|\log (|x|)| \\
& \geq \frac{c_{43}}{2} k|x|^{-N+2 \alpha} \geq c_{46} \frac{|x|^{-\frac{p(N-2 \alpha)}{p-1}}}{(1+|\log (|x|)|)^{\frac{1}{p-1}}},
\end{aligned}
$$

where $c_{46}=\frac{1}{2} c_{43} c_{45}$ is independent of $k$.

By (4.25), we can choose a sequence $k_{n} \in[1,+\infty)$ such that

$$
\rho_{k_{n+1}} \geq \frac{1}{2} \rho_{k_{n}},
$$

Then for any $x \in B_{\rho_{k_{0}}} \backslash\{0\}$, there exists $k_{n}$ such that $x \in B_{\rho_{k_{n}}} \backslash \frac{\rho_{\rho_{k_{n}}}}{2}$. By (4.26) there holds

$$
u_{k_{n}}(x) \geq c_{46} \frac{|x|^{-\frac{p(N-2 \alpha)}{p-1}}}{(1+|\log (|x|)|)^{\frac{1}{p-1}}}
$$


Together with $u_{k+1}>u_{k}$, we infer

$$
u_{\infty}(x) \geq c_{46} \frac{|x|^{-\frac{p(N-2 \alpha)}{p-1}}}{(1+|\log (|x|)|)^{\frac{1}{p-1}}} \quad \forall x \in B_{\rho_{k_{0}}} \backslash\{0\} .
$$

(iii) By (1.9) and Lemma 2.1-(iii) with $p \in\left(1+\frac{2 \alpha}{N}, \frac{2 \alpha}{N-2 \alpha}\right)$, there exist $\rho_{0} \in\left(0, r_{0}\right)$ and $c_{47}, c_{48}>0$ such that

$$
u_{k}(x) \geq c_{47} k|x|^{-N+2 \alpha}-c_{48} k^{p} \quad \forall x \in B_{\rho_{0}} \backslash\{0\} .
$$

Put

$$
\rho_{k}=\left(\frac{c_{48}}{2 c_{47}} k^{p-1}\right)^{-\frac{1}{N-2 \alpha}},
$$

then $\lim _{k \rightarrow \infty} \rho_{k}=0$ and there exists $k_{0}>0$ such that $\rho_{k_{0}} \leq \rho_{0}$. Therefore, if $x \in B_{\rho_{k}} \backslash B_{\frac{\rho_{k}}{2}}$ and $k \geq k_{0}$, there holds

$$
c_{48} k^{p}=\frac{c_{47}}{2} k \rho_{k}^{-N+2 \alpha} \leq \frac{c_{47}}{2} k|x|^{-N+2 \alpha},
$$

which yields

$$
k=\left(\frac{c_{48}}{2 c_{47}}\right)^{-\frac{1}{p-1}} \rho_{k}^{-\frac{N-2 \alpha}{p-1}} \geq c_{49}|x|^{-\frac{N-2 \alpha}{p-1}},
$$

by (4.28), where $c_{49}=2^{-\frac{N-2 \alpha}{p-1}}\left(\frac{c_{48}}{2 c_{47}}\right)^{-\frac{1}{p-1}}$. Consequently,

$$
\begin{aligned}
u_{k}(x) \geq c_{47} k|x|^{-N+2 \alpha}-c_{48} k^{p} & \geq \frac{c_{47}}{2} k|x|^{-N+2 \alpha} \\
& \geq c_{50}|x|^{-\frac{p}{p-1}(N-2 \alpha)},
\end{aligned}
$$

where $c_{50}=\frac{1}{2} c_{47} c_{49}$ is independent of $k$.

By (4.28), we can choose a sequence $k_{n} \in[1,+\infty)$ such that

$$
\rho_{k_{n+1}} \geq \frac{1}{2} \rho_{k_{n}},
$$

Then for any $x \in B_{\rho_{k_{0}}} \backslash\{0\}$, there exists $k_{n}$ such that $x \in B_{\rho_{k_{n}}} \backslash B \frac{\rho_{k_{n}}}{2}$ and then by (4.29),

$$
u_{k_{n}}(x) \geq c_{50}|x|^{-\frac{p(N-2 \alpha)}{p-1}} .
$$

Together with $u_{k+1}>u_{k}$, we have

$$
u_{\infty}(x) \geq c_{50}|x|^{-\frac{p(N-2 \alpha)}{p-1}} \quad \forall x \in B_{\rho_{k_{0}}} \backslash\{0\},
$$

which ends the proof. 
Lemma 4.2 Let $p \in\left(1+\frac{2 \alpha}{N}, p_{\alpha}^{*}\right)$ and $u_{s}$ be a strongly singular solution of (1.4) satisfying (1.5). Then

$$
u_{\infty} \leq u_{s} \text { in } \Omega \backslash\{0\},
$$

where $u_{\infty}$ is defined by (1.13).

Proof. By (1.5) and (1.9), it follows

$$
\lim _{x \rightarrow 0} u_{s}|x|^{\frac{2 \alpha}{p-1}}=c_{0} \quad \text { and } \quad \lim _{x \rightarrow 0} u_{k}|x|^{N-2 \alpha}=c_{k},
$$

which implies that there exists $r_{1}>0$ such that

$$
u_{k}<u_{s} \text { in } B_{r_{1}} \backslash\{0\} .
$$

Since by Theorem 3.1, $u_{k}$ satisfies

$$
(-\Delta)^{\alpha} u_{k}+u_{k}^{p}=0 \quad \text { in } \quad \Omega \backslash B_{r_{1}}(0),
$$

so does $u_{s}$. By Theorem 2.1 there holds $u_{k} \leq u_{s}$ in $\Omega \backslash\{0\}$. Jointly with (1.13), it implies

$$
u_{\infty} \leq u_{s} \quad \text { in } \quad \Omega \backslash\{0\}
$$

Proof of Theorem 1.1 (ii) and Theorem 1.2 (iv). By Lemma 4.2 and Theorem 3.2, we obtain that $u_{\infty}$ is a classical solution of (1.4). Moreover, by Proposition 4.5 part $(i)$ and Lemma 4.2, we have

$$
\frac{1}{c_{51}}|x|^{-\frac{2 \alpha}{p-1}} \leq u_{\infty}(x) \leq c_{51}|x|^{-\frac{2 \alpha}{p-1}}
$$

for some $c_{51}>1$. Then $u_{\infty}=u_{s}$ in $\mathbb{R}^{N} \backslash\{0\}$ since $u_{s}$ is unique in the class of solutions satisfying (1.6).

\subsection{Proof of Theorem $1.2(i i)$ and (iii)}

By Lemma 4.2 and Theorem 3.2, $u_{\infty}$ is a classical solution of (1.4) and it satisfies

$$
u_{\infty} \leq u_{s} \quad \text { in } \quad \Omega \backslash\{0\} .
$$

Therefore (1.16) and (1.15) follow by Proposition 4.5 part (ii) and (iii), respectively. 


\section{References}

[1] Ph. Benilan and H. Brezis, Nonlinear problems related to the ThomasFermi equation, J. Evolution Eq. 3, 673-770, (2003).

[2] H. Brezis, Some variational problems of the Thomas-Fermi type. Variational inequalities and complementarity problems, Proc. Internat. School, Erice, Wiley, Chichester, 53-73 (1980).

[3] H. Brezis and L. Véron, Removable singularities of some nonlinear elliptic equations, Arch. Rational Mech. Anal. 75, 1-6 (1980).

[4] L. Caffarelli and L. Silvestre, Regularity theory for fully non-linear integrodifferential equations, Comm. Pure Appl. Math. 62, 597-638 (2009).

[5] H. Chen, P. Felmer and A. Quaas, Large solutions to elliptic equations involving fractional Laplacian, submitted.

[6] H. Chen and L. Véron, Solutions of fractional equations involving sources and Radon measures, HAL : hal-00766824, version 1, Dec. 2012.

[7] H. Chen and L. Véron, Singular solutions of fractional elliptic equations with absorption, arXiv:1302.1427v1, [math.AP], 6 (Feb. 2013).

[8] H. Chen and L. Véron, Semilinear fractional elliptic equations involving measures, arXiv:1305.0945v2, [math.AP], 15 (May 2013).

[9] Z. Chen, and R. Song, Estimates on Green functions and poisson kernels for symmetric stable process, Math. Ann. 312, 465-501 (1998).

[10] X. Ros-Oton and J. Serra, The Dirichlet problem for the fractional laplacian: regularity up to the boundary, J. Math. Pures Appl., to appear.

[11] L. Silvestre, Regularity of the obstacle problem for a fractional power of the laplace operator, Comm. Pure Appl. Math. 60, 67-112 (2007).

[12] L. Véron, Weak and strong singularities of nonlinear elliptic equations, Proc. Symp. Pure Math. 45, 477-495 (1986).

[13] L. Véron, Singular solutions of some nonlinear elliptic equations, Nonlinear Anal. T. M. \& A. 5, 225-242 (1981).

[14] L. Véron, Elliptic equations involving Measures, Stationary Partial Differential equations, Vol. I, 593-712, Handb. Differ. Equ., NorthHolland, Amsterdam (2004). 